\title{
Ibuprofen Treatment for Closure of Patent Ductus Arteriosus Is Not Associated With Increased Risk of Neuropathology
}

\author{
MICHELLE LOELIGER, AMY SHIELDS, DONALD MCCURNIN, RONALD I. CLYMAN, BRADLEY YODER, TERRIE E. INDER, \\ AND SANDRA M. REES
}

Department of Anatomy and Cell Biology [M.L., A.S., S.M.R.], University of Melbourne, Melbourne, Victoria 3010, Australia; Department of Pediatrics [D.M.], The University of Texas Southwestern Medical Center at Dallas, Dallas, Texas 75390; Department of Pediatrics [R.I.C.], University of California, San Francisco, California 94143; Department of Pediatrics [B.Y.], University of Utah, Salt Lake City, Utah 84108; Department of Pediatrics [T.E.I.], Washington University, St Louis, Missouri 63130

\begin{abstract}
Ibuprofen is an effective pharmacological intervention for closure of a patent ductus arteriosus (PDA) in preterm infants and is an alternative to surgical ligation; however, it is not certain whether ibuprofen treatment is associated with adverse effects on the brain. Therefore, this study examined neuropathological outcomes of ibuprofen therapy for a PDA. Fetal baboons were delivered at $125 \mathrm{~d}$ of gestation ( $\mathrm{dg}$; term $\sim 185 \mathrm{dg}$ ) by caesarean section, given surfactant, and ventilated for $14 \mathrm{~d}$ with positive pressure ventilation (PPV). Baboons were randomly allocated to receive either ibuprofen (PPV+ ibuprofen, $n=8$ ) or no therapy (PPV, $n=5$ ). Animals were killed on day 14 and brains assessed for cerebral growth, development, and neuropathology. Body and brain weights, the total volume of the brain, and the surface folding index (measure of brain growth) were not different $(p>0.05)$ between PPV + ibuprofen-treated and PPV animals. There was no difference $(p>0.05)$ in the number of myelin basic protein-immunoreactive (IR) oligodendrocytes, glial fibrillary acid protein-IR astrocytes, or Iba1-IR macrophages/microglia in the forebrain. No overt cerebellar alterations were observed in either group. Ibuprofen treatment for PDA closure in the preterm baboon neonate is not associated with any increased risk of neuropathology or alterations to brain growth and development. (Pediatr Res 68: 298-302, 2010)
\end{abstract}

$S^{n-1}$ urvival of prematurely delivered and low birth weight infants has improved in recent years because of the advent of improved prenatal and neonatal care strategies. However, these infants are at greater risk of poorer neurological outcomes than their full-term counterparts, with $\sim 10 \%$ likely to develop cerebral palsy, while sensory and motor impairments and developmental delays are observed in 10 to $20 \%$ of infants (1-3). There is consequently considerable interest in the role that postnatal management and intervention strategies may play in neurological development.

Hemodynamic symptoms from a patent ductus arteriosus (PDA) are present in 55 to $70 \%$ of infants delivered $<1000 \mathrm{~g}$ or before 28 wk of gestation, with the PDA resulting in alterations in cerebral, renal, and mesenteric perfusion in addition to producing pulmonary edema, impairing pulmonary

Received March 24, 2010; accepted May 28, 2010.

Correspondence: Michelle Loeliger, Ph.D., Department of Anatomy and Cell Biology, University of Melbourne, Victoria, Melbourne 3010, Australia; e-mail: m.loeliger@unimelb.edu.au

Supported by NIH Grant R01 HL074942 and in part by NIH Grants HL52636, HL56061, HL46691, HL77395, and HL52646. mechanics, increasing the risk of pulmonary hemorrhage and prolonging the need for mechanical ventilation (4). A persistent PDA is associated with the development of bronchopulmonary dysplasia (BPD); however, its direct role in causing BPD is not known (5-7).

Current therapies for a PDA include pharmacological treatment with indomethacin or ibuprofen and/or surgical ligation. Surgical ligation produces definitive closure of a PDA, but there is controversy as to its effects on subsequent neurodevelopmental outcome $(5,7)$. The trauma of surgery and the possible adverse effects of anesthetics on the brain (8) are also factors to consider. Ibuprofen and indomethacin are effective treatments for closure; however, only a few studies have investigated whether there are any neuropathological or functional sequelae (9-13).

Assessment of such outcomes in humans is likely to be complicated by the unique and varying treatments that each infant experiences while in the neonatal intensive care unit (NICU) setting. We have the unique opportunity to examine neurological development in a baboon model, prematurely delivered at $125 \mathrm{~d}$ of gestation $(\mathrm{dg})$, which has comparable brain (14) and cardiopulmonary $(15,16)$ development to human preterm infants at 26 to $27 \mathrm{wk}$ gestation. These baboons are maintained in a NICU setting that is similar to that used for premature human infants. Therefore, we have an appropriate model in which to investigate the influence of postnatal interventional strategies that have relevance to prematurely delivered human infants.

Premature baboons have a similar neonatal course to human infants, developing respiratory distress and failure of PDA closure after birth. They develop histopathological changes in the lung similar to those described for human infants with BPD despite antenatal glucocorticoid treatment, early postnatal surfactant replacement, low tidal volume ventilation, and low supplemental oxygen administration during the first 14-d postdelivery $(15,16)$. Pharmacological closure with ibuprofen improves pulmonary mechanics and decreases the detrimental

Abbreviations: BPD, bronchopulmonary dysplasia; $\mathbf{F i O}_{2}$, fraction of inspired oxygen; GFAP, glial fibrillary acidic protein; MAP, mean arterial pressure; PDA, patent ductus arteriosus; PPV, positive pressure ventilation; SFI, surface folding index 
effects of preterm birth on alveolarization (17). Because the impact of ibuprofen on the immature nonhuman primate brain is not known, our objective in this study was to examine brain growth and development in our premature baboon model of neonatal chronic lung disease (CLD) after pharmacological treatment of the PDA with ibuprofen commencing at $24 \mathrm{~h}$ of age. We hypothesized that ibuprofen treatment will not increase the risk of neuropathology or impair brain growth when compared with no treatment.

\section{METHODS}

All animal studies were performed at the Southwest Foundation for Biomedical Research in San Antonio, TX. All animal husbandry, animal handling, and procedures were reviewed and approved to conform to American Association for Accreditation of Laboratory Animal Care guidelines.

Delivery and instrumentation. Pregnant baboon dams (Papio papio) with timed gestations were treated with $6 \mathrm{mg}$ of i.m. administered betamethasone 48 and $24 \mathrm{~h}$ before elective delivery at $125 \pm 2 \mathrm{dg}$ (term $\sim 185 \mathrm{dg}$ ). At birth, animals were weighed, sedated, intubated, and treated with $4 \mathrm{~mL} / \mathrm{kg}$ surfactant (Survanta; Courtesy Ross Laboratories, Columbus, $\mathrm{OH}$ ) before the initiation of ventilatory support.

Ventilatory management and hemodynamic change. Newborn baboons were mechanically ventilated for $14 \mathrm{~d}$. A complete description of the details of the surgical procedures and animal care (including ventilator management, target goals for $\mathrm{PaO}_{2}, \mathrm{PaCO}_{2}$, tidal volume, and nutritional, fluid, transfusion, antibiotic, and blood pressure management) have been described previously (17). Animals were randomized before delivery to either receive ibuprofen [positive pressure ventilation (PPV) + ibuprofen, $n=8$ ] or no treatment (PPV, $n=5$ ). Ibuprofen was administered i.v. ( $>20 \mathrm{~min}$ ) according to the following schedule: $10 \mathrm{mg} / \mathrm{kg}(24 \mathrm{~h}), 5 \mathrm{mg} / \mathrm{kg}$ ( $48 \mathrm{~h}$ ), $5 \mathrm{mg} / \mathrm{kg}(72 \mathrm{~h}), 5 \mathrm{mg} / \mathrm{kg}$ (96 h), and $5 \mathrm{mg} / \mathrm{kg}(120 \mathrm{~h})$.

Tissue collection and processing. At necropsy (at $14 \mathrm{~d}$ ), brains were weighed and immersed in $4 \%$ paraformaldehyde in $0.1 \mathrm{M}$ phosphate buffer, and coronal blocks from the right forebrain (at 5-mm intervals) and a mid-sagittal block from the cerebellar vermis of each brain were processed to paraffin. Ten, $8-\mu \mathrm{m}$ sections were cut from the rostral surface of each forebrain block (10 to 12 per animal) and in the sagittal plane for the cerebellum.

A section from each block was stained with hematoxylin and eosin (H\&E) and assessed qualitatively for gross morphological changes including lesions and the presence of hemorrhages (scored: present, 1; absent, 0). Masson's trichrome was used to assess for collagen deposition; van Gieson's stain for elastic fibers; reticulin for reticulated fibers; and Perls stain to visualize hemosiderin deposition.

Rabbit anti-rat calbindin (1:500; Swant, Bellinzona, Switzerland) was used to identify cerebellar Purkinje cells; rabbit anti-cow glial fibrillary acidic protein (GFAP; 1:500; Sigma Chemical, Co., St. Louis, MO) to identify astrocytes; rat anti-bovine myelin basic protein (MBP, 1:100; Chemicon International, $\mathrm{CA}$ ) to assess myelination; rabbit anti-ionized calcium-binding adapter molecule 1 (Iba1; 1:1500; Wako Chemicals, Richmond, VA) to identify microglia/macrophages, as described previously (18). Control and experimental material were stained simultaneously to avoid procedural variation. There was no staining when the primary antibody was omitted.

Morphological analysis. Analyses were performed on a section from each block for all brains of PPV + ibuprofen and PPV animals unless otherwise stated; measurements were made on coded slides. Areas and widths were assessed using a digitizing program (Sigma Scan Pro v4, SPSS Science, Chicago, IL), and counts were performed using an image analysis system (Image Pro Plus v4.1; Media Cybernetics, MD). All measurements were performed on all animals; means were calculated for each animal, and then a group mean was determined.

Qualitative analysis. A thorough qualitative assessment of each brain was performed in each block including all regions (cerebral hemispheres including white matter, neocortex, basal ganglia, thalamus, hippocampus, and cerebellum) by two examiners. Sections were assessed for presence of lesions, neuronal death and focal gliosis (astrogliosis or microgliosis), and glial and microglial invasion.

Quantitative analysis. In H\&E-stained sections, the volumes of white matter, neocortex, deep gray matter (basal ganglia plus thalamus), hippocampus, and ventricles were estimated by measuring the cross-sectional area of each region in a section from each block (10 to 12 per animal) and applying the Cavalieri principle (18).
In H\&E-stained sections from each block, the surface folding index (SFI), which gives an estimation of the expansion of the surface area relative to volume, was determined (19).

Point counting (20) was used to determine the percentage of white matter occupied by blood vessel profiles in deep and subcortical white matter $(\times 660)$ as an indicator of vasodilation or vasculogenesis. Assessment was performed in GFAP-immunoreactive (IR) sections at each level $(\sim 12$ sections, two regions in each section, and 24 measurements/region in total) as blood vessel profiles are clearly delineated (21).

GFAP-IR cells were counted $(\times 660)$ in two randomly selected areas $(0.02$ $\left.\mathrm{mm}^{2}\right)$ in each of the deep and subcortical white matter regions $(\sim 12$ sections/ animal, 24 measurements/region in total); the cerebral neocortex (three sites in blocks from frontal/temporal, parietal/temporal, and occipital lobes in layers 5 and 6); and the hippocampus (stratum radiatum in the CA1 region, two sections/animal and four measurements in total).

MBP-IR oligodendrocytes were counted $(\times 300)$ in two sections per animal. Two regions $\left(0.42 \mathrm{~mm}^{2}\right)$ in each section were randomly selected in both the deep and subcortical white matter from the parietal/temporal lobe (four measurements/region in total).

Iba1-IR cells were counted $(\times 660)$ in randomly selected areas $\left(0.02 \mathrm{~mm}^{2}\right)$ in each of the deep and subcortical white matter regions (three sites in blocks from each of the frontal/temporal, parietal/temporal, and occipital lobes and six measurements/region in total).

In H\&E-stained sections, the width of the external granule layer (EGL) was assessed in the cerebellum (22) in 10 randomly selected regions in each of lobules 1 and 8 (three measurements/region and 60 measurements/animal in total).

Semiquantitative analysis. GFAP-IR radial glial fibers in the forebrain were scored on a scale of 0-3 (19).

Physiological data. Physiological data, including arterial blood gases $\left[\mathrm{PaO}_{2}, \mathrm{PO}_{2}, \mathrm{PaCO}_{2}, \mathrm{PCO}_{2}, \mathrm{pH}\right.$, and fraction of inspired oxygen $\left.\left(\mathrm{FiO}_{2}\right)\right]$, mean arterial blood pressure (MAP), and heart rate (HR), were monitored throughout the experimental period, and mean values were calculated. The "interval flux" of physiological parameters was calculated as a surrogate measure of instability as described previously (23). The interval flux for a specific physiological variable was the difference between the maximum and minimum values of the variable during a specified time interval (23). For each animal we then 1) identified the maximum flux and 2) calculated the mean of the interval fluxes during the entire experimental period. In previous studies, a greater degree of flux particularly in $\mathrm{FiO}_{2}$ has been associated with an increased incidence of neuropathology $(19,23-25)$.

Statistical analysis. Linear regression analysis was performed to determine whether there was a correlation between a) physiological variables and quantitative parameters and b) quantitative parameters and volumetric measurements. Significance of differences between PPV + ibuprofen and PPV groups was tested using $t$ tests; a probability of $p<0.05$ was considered to be significant. Results are expressed as mean \pm SEM (weights and areas) and mean of means \pm SEM (histological parameters).

\section{RESULTS}

Ventilatory management and hemodynamic change. The ductus in the PPV + ibuprofen group either closed and remained closed, $n=5$, or closed and then intermittently reopened, $n=3$, after PPV + ibuprofen treatment. In contrast, all the animals in the PPV group had a PDA that remained open throughout the entire 14-d experiment [mean pulmonary to systemic blood flow (Qp/Qs) ratio from $24 \mathrm{~h}$ through $14 \mathrm{~d}$ PPV, $1.8 \pm 0.2$ versus PPV+ ibuprofen, $1.1 \pm 0.1 ; p<$ $0.001]$. The PPV + ibuprofen group had a significantly higher $(p<0.05)$ mean (in addition to systolic and diastolic) systemic blood pressure after the start of treatment. There were no differences $(p>0.05)$ between the two groups in base deficit, serum bicarbonate, or need for dopamine/dobutamine administration during the 14-d treatment course. There were also no differences $(p>0.05)$ in the fluid intake and urine output between the two groups (17).

Brain growth and development. There was no difference $(p>0.05)$ between groups in the body, brain, and cerebellar weights or brain/body weight ratio (Table 1). 
Table 1. Body and brain weights and volumetric measurements

\begin{tabular}{lcc}
\hline \multicolumn{1}{c}{ Parameter } & PPV $(n=5)$ & $\begin{array}{c}\text { PPV }+ \\
\text { ibuprofen } \\
(n=8)\end{array}$ \\
\hline Body weight at birth $(\mathrm{g})$ & $367 \pm 7$ & $360 \pm 20$ \\
Body weight at necropsy $(\mathrm{g})$ & $339 \pm 11$ & $355 \pm 17$ \\
Total brain weight $(\mathrm{g})$ & $44.3 \pm 2.0$ & $45.4 \pm 1.0$ \\
Cerebellar weight $(\mathrm{g})$ & $1.4 \pm 0.1$ & $1.5 \pm 0.1$ \\
Brain/body weight ratio & $0.123 \pm 0.005$ & $0.135 \pm 0.004$ \\
Volume of right forebrain $\left(\mathrm{mm}^{3}\right)$ & $13,771 \pm 851$ & $14,319 \pm 525$ \\
Ventricular volume $\left(\mathrm{mm}^{3}\right)$ & $232 \pm 33$ & $277 \pm 35$ \\
White matter volume $\left(\mathrm{mm}^{3}\right)$ & $5,867 \pm 339$ & $6,070 \pm 272$ \\
Neocortical volume $\left(\mathrm{mm}^{3}\right)$ & $6,574 \pm 656$ & $6,576 \pm 374$ \\
Deep grey matter & $1,195 \pm 71$ & $1,261 \pm 90$ \\
$\quad$ basal ganglia, thalamus) & & \\
$\quad$ volume (mm $\left.{ }^{3}\right)$ & $109 \pm 18$ & $149 \pm 24$ \\
Hippocampal volume $\left(\mathrm{mm}^{3}\right)$ & $1.7 \pm 0.3$ & $2.0 \pm 0.3$ \\
Ventricular/total volume $(\%)$ & $42.7 \pm 0.9$ & $42.5 \pm 1.4$ \\
White matter/total volume $(\%)$ & $47.3 \pm 2.1$ & $45.8 \pm 1.7$ \\
Neocortex/total volume $(\%)$ & $8.8 \pm 0.5$ & $8.8 \pm 0.7$ \\
Deep grey matter/total volume $(\%)$ & $0.8 \pm 0.1$ & $1.0 \pm 0.1$ \\
Hippocampal/total volume $(\%)$ & $1.1 \pm 0.1$ & $1.1 \pm 0.1$ \\
White matter/neocortex ratio & $41.7 \pm 2.7$ & $41.2 \pm 1.5$ \\
SFI & & \\
\hline
\end{tabular}

Values are mean \pm SEM. All volume measurements were made on the right hemisphere only.

There was no difference $(p>0.05)$ between groups in the total volume of the forebrain (right), ventricles, white matter, neocortical, deep gray matter, or hippocampal volumes (Table 1). There was no difference $(p>0.05)$ between the groups in the ratios of ventricles, white matter, neocortical, or deep gray matter volumes to the total forebrain volume nor in the ratio of white matter/neocortex (Table 1).

The overall SFI of the forebrain was not different $(p>0.05)$ between groups (Table 1).

Qualitative assessment of brain injury. There was no evidence of cerebral infarction or hemorrhages (intraventricular, intracerebral, or subventricular) in any animal in either group. There was also no evidence of cystic or noncystic lesions, neuronal loss (pyknosis or apoptosis), hypertrophic astrocytes, or focal aggregates of astroglia or microglia in either group in any region of the forebrain including the white matter, neocortex, hippocampus and deep gray matter (basal ganglia and thalamus), or the cerebellum.

Quantitative assessment of the brain. There was no difference $(p>0.05)$ between groups in either the deep or subcortical white matter, cerebral neocortex, or the stratum radiatum of the hippocampus (Table 2; Fig. 1).

The overall density in both the deep and subcortical white matter was not different $(p>0.05)$ between groups (Table 2; Fig. 1).

There was no difference $(p>0.05)$ between ventilated groups in the number of ramified Iba1-IR cells in the deep and subcortical white matter or within the neocortex (Table 2; Fig. 1). Activated microglia (round morphology and attenuated processes) were observed infrequently in both groups.

Semiquantitative assessment of brain injury. Intensely GFAP-IR radial glial fibers were present at the ventricular
Table 2. Forebrain parameters

\begin{tabular}{lcc}
\hline \multicolumn{1}{c}{ Parameter } & $\begin{array}{c}\text { PPV }+ \\
\text { PPuprofen } \\
(n=8)\end{array}$ \\
\hline Astrocytes & $594 \pm 39$ & $589 \pm 28$ \\
$\quad$ Deep white matter (cells $\left./ \mathrm{mm}^{2}\right)$ & $563 \pm 20$ & $592 \pm 35$ \\
$\quad$ Subcortical white matter & & \\
$\quad\left(\right.$ cells $\left./ \mathrm{mm}^{2}\right)$ & $323 \pm 40$ & $342 \pm 27$ \\
Neocortex $\left(\right.$ cells $\left./ \mathrm{mm}^{2}\right)$ & $616 \pm 73$ & $672 \pm 36$ \\
Hippocampus $\left(\right.$ cells $\left./ \mathrm{mm}^{2}\right)$ & & \\
MBP-IR oligodendrocytes & $327 \pm 31$ & $249 \pm 25$ \\
$\quad$ Deep white matter $\left(\right.$ cells $\left./ \mathrm{mm}^{2}\right)$ & $81 \pm 15$ & $80 \pm 29$ \\
Subcortical white matter & & \\
$\quad\left(\right.$ cells $\left./ \mathrm{mm}^{2}\right)$ & & \\
Iba1-IR microglia/macrophages & $189.2 \pm 19.9$ & $200.7 \pm 24.5$ \\
Deep white matter $\left(\right.$ cells $\left./ \mathrm{mm}^{2}\right)$ & & $114 \pm 13$ \\
Subcortical white matter & & \\
$\quad\left(\right.$ cells $\left./ \mathrm{mm}^{2}\right)$ & $54.0 \pm 12.0$ & $52.9 \pm 5.2$ \\
$\quad$ Neocortex $\left(\right.$ cells $\left./ \mathrm{mm}^{2}\right)$ & $2.5 \pm 0.2$ & $2.5 \pm 0.2$ \\
GFAP-IR radial glial fibres & & \\
\hline
\end{tabular}

Values are mean \pm SEM.
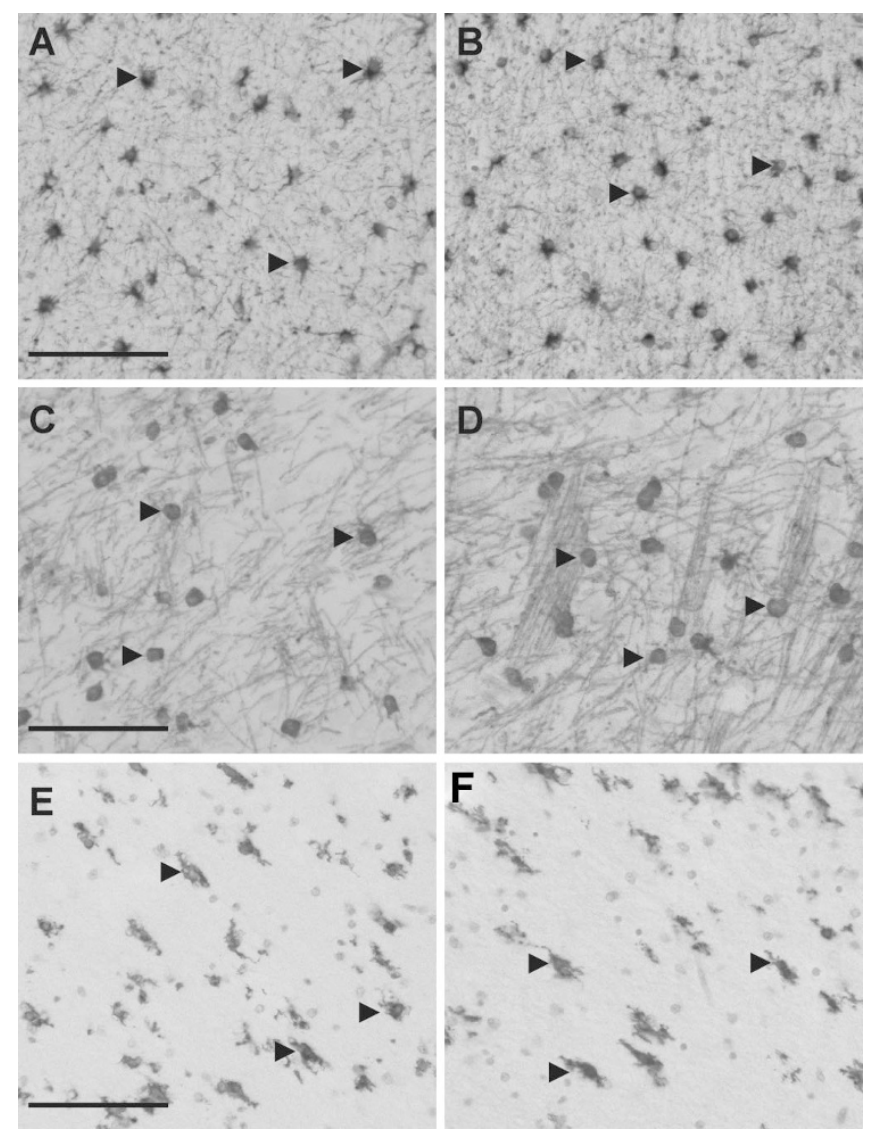

Figure 1. There was no difference in the areal density of GFAP-IR astrocyte in the deep white matter between PPV $(A)$ and PPV + ibuprofen $(B)$ animals; arrows indicate astrocytes. The areal density of MBP-IR oligodendrocytes in cerebral WM was not different between PPV $(C)$ and PPV+ ibuprofen $(D)$ animals; arrows indicate MBP-IR oligodendrocytes. The areal density of Iba-IR macrophages/microglia (arrows) in the cerebral WM was not different between PPV $(E)$ and PPV+ ibuprofen $(F)$ animals. Scale bars $A-F=100$ $\mu \mathrm{m}$; WM, white matter.

surface projecting into the deep white matter in all animals. There was no difference $(p>0.05)$ in the occurrence of GFAP-IR radial glia between groups (Table 2). 
Table 3. Mean interval flux

\begin{tabular}{lcc}
\hline \multicolumn{1}{c}{ Parameter } & $\begin{array}{c}\text { PPV } \\
(n=5)\end{array}$ & $\begin{array}{c}\text { PPV }+ \text { ibuprofen } \\
(n=8)\end{array}$ \\
\hline $\mathrm{pH}$ & $0.15 \pm 0.01$ & $0.13 \pm 0.02$ \\
$\mathrm{PaO}_{2}(\mathrm{~mm} \mathrm{Hg})$ & $33.4 \pm 1.2^{*}$ & $25.4 \pm 2.7$ \\
$\mathrm{PaCO}_{2}(\mathrm{~mm} \mathrm{Hg})$ & $22.3 \pm 2.1$ & $16.1 \pm 1.7$ \\
$\mathrm{FiO}_{2}$ & $0.12 \pm 0.01$ & $0.09 \pm 0.02$ \\
$\mathrm{MAP}$ & $9.0 \pm 0.6$ & $9.9 \pm 0.6$ \\
$\mathrm{HR}$ & $18.2 \pm 0.6$ & $18.8 \pm 1.1$ \\
\hline
\end{tabular}

Values are mean interval flux \pm SEM.

$* p<0.05$ vs PPV + ibuprofen.

Cerebellum. No hemorrhages, regions of infarction, or overt structural anomalies were observed in either group. There was no difference $(p>0.05)$ in the width of the EGL between groups $(\mathrm{PPV}+$ ibuprofen, $31.8 \pm 1.6 \mathrm{~mm}$ versus PPV, $33.1 \pm 2.5 \mathrm{~mm}$ ).

Physiological responses. There was an increase $(p<0.05)$ in the mean interval flux of $\mathrm{PaO}_{2}$ in PPV when compared with $\mathrm{PPV}+$ ibuprofen-treated animals. There was no difference $(p>0.05)$ in the mean interval flux of $\mathrm{pH}, \mathrm{PaCO}_{2}, \mathrm{FiO}_{2}, \mathrm{MAP}$, or HR between groups during the 14-d study period (Table 3).

Relationship of brain injury to brain volume and physiology. There was no correlation $(p>0.05)$ between physiological variables $\left(\mathrm{pH}, \mathrm{PaO}_{2}, \mathrm{PaCO}_{2}, \mathrm{FiO}_{2}, \mathrm{MAP}\right.$, and $\left.\mathrm{HR}\right)$ and quantitative parameters (volumetric measurements and astrocyte and oligodendrocyte densities).

There was a negative correlation between the total volume of white matter and the number of astrocytes in the deep white matter $\left(r^{2}=0.48 ; p<0.01\right)$ and a positive correlation between the total volume of white matter and the SFI $\left(r^{2}=\right.$ $0.34 ; p<0.04)$. This indicates that an increase in gliosis is associated with a decrease in white matter growth and that increasing white matter volume is associated with increased gyral formation.

\section{DISCUSSION}

The major finding of this study is that pharmacological closure of a PDA with ibuprofen does not pose an increased risk of brain injury and/or impairment in brain development compared with no treatment in the first days of life. There was no difference between treated or untreated animals in brain weight, gyral formation, or relative growths of white and gray matter. Nor was there a difference in astrocyte, oligodendrocyte, or microglial densities in any region of the brain suggesting that ibuprofen treatment does not cause astrogliosis, affect the oligodendrocyte lineage and myelination, or have an impact on the cerebral inflammatory response. Data from gestational control animals have not been included here as we have already established in previous studies using this model $(19,23-25)$ that, premature delivery per se reduces the normal trajectory of brain growth and increases the incidence of subtle neuropathologies; the purpose of this study was to examine the difference between treatment groups. We cannot directly compare the neuropathological outcomes from this study where animals received antenatal steroids with our earlier study on ductal ligation where steroids were not ad- ministered (23); however, we note that neither regimen was associated with overt damage such as hemorrhage or cystic infarction.

It is possible that our findings could be viewed from a different perspective. In the study alluded to above (23), we found that animals exposed to a persistent PDA had subtle increases in neuropathology and a trend toward poorer brain growth compared with animals with ductal ligation. If the PDA does contribute to adverse effects on the premature brain, then one might anticipate that its effective closure with ibuprofen should have resulted in fewer astrocytes, increased density of oligodendrocytes, and better cerebral growth. This was not observed in our study.

It is possible that the use of antenatal glucocorticoids, in this study, minimizes the differences between the two treatment groups. It is also possible that the lack of difference indicates that any benefit derived from PDA closure was negated by ibuprofen. We think the second alternative is less likely because pharmacological treatment of PDA with cyclooxygenase inhibitors has been associated with better, not worse, neurological outcomes in preterm infants. Prophylactic indomethacin has been associated with a decreased risk for periventricular and intraventricular hemorrhage (IVH) $(11,13)$ and seems to have no adverse effects on later neurodevelopmental outcome (11-13). Furthermore, early ibuprofen treatment has been associated with a reduction in periventricular leukomalacia (9). Thus, our findings concur with data from these preterm human infant studies demonstrating that early closure of a patent PDA compared with no treatment is not associated with any increase in significant neuropathology nor does it increase the risk of altered brain growth and development. It is reassuring that no novel form of brain injury or alteration in brain development was noted in the setting of ibuprofen treatment.

In translating our findings to the human preterm infant, we acknowledge that there are limitations in our study including the small number of animals and the relatively short duration of the study. We also note that the preterm baboon model was electively delivered without any preexisting complications such as infection, hypoxemia, or growth restriction. Although the long-term effects of ibuprofen treatment on the brain cannot be established in this study, it is likely that pharmacological closure may provide benefits if the PDA is contributing to persistent pulmonary and hemodynamic instability as has been shown in other organ systems (26).

\section{CONCLUSIONS}

These findings show that ibuprofen treatment for PDA closure in the preterm baboon neonate is not associated with any increased risk of neuropathology and/or alterations to brain growth and development. Furthermore, this therapy has been shown to improve pulmonary outcomes (17).

Acknowledgments. We thank Dr. Jacqueline Coalson, Ms. Vicki Winter, and staffs at the Bronchopulmonary Dysplasia Resource Centre, San Antonio, TX, for provision of baboon tissue. 


\section{REFERENCES}

1. Bodeau-Livinec F, Marlow N, Ancel PY, Kurinczuk JJ, Costeloe K, Kaminski M 2008 Impact of intensive care practices on short-term and long-term outcomes for extremely preterm infants: comparison between the British Isles and France. Pediatrics 122:e1014-e1021

2. Marlow N, Wolke D, Bracewell MA, Samara M 2005 Neurologic and developmental disability at six years of age after extremely preterm birth. N Engl J Med 352:9-19

3. Marlow N, Hennessy EM, Bracewell MA, Wolke D 2007 Motor and executive function at 6 years of age after extremely preterm birth. Pediatrics 120:793-804

4. Clyman RI 2006 Mechanisms regulating the ductus arteriosus. Biol Neonate 89:330-335

5. Chorne N, Leonard C, Piecuch R, Clyman RI 2007 Patent ductus arteriosus and its treatment as risk factors for neonatal and neurodevelopmental morbidity. Pediatrics 119:1165-1174

6. Clyman RI, Chorne N 2007 Patent ductus arteriosus: evidence for and against treatment. J Pediatr 150:216-219

7. Kabra NS, Schmidt B, Roberts RS, Doyle LW, Papile L, Fanaroff A; Trial of Indomethacin Prophylaxis in Preterms Investigators 2007 Neurosensory impairment after surgical closure of patent ductus arteriosus in extremely low birth weight infants: results from the Trial of Indomethacin Prophylaxis in Preterms. J Pediatr 150:229-234, 234.e1

8. Creeley CE, Olney JW 2010 The young: neuroapoptosis induced by anesthetics and what to do about it. Anesth Analg 110:442-448

9. Aranda JV, Clyman R, Cox B, Van Overmeire B, Wozniak P, Sosenko I, Carlo WA, Ward RM, Shalwitz R, Baggs G, Seth A, Darko L 2009 A randomized, double-blind, placebo-controlled trial on i.v. ibuprofen L-lysine for the early closure of nonsymptomatic patent ductus arteriosus within 72 hours of birth in extremely low-birthweight infants. Am J Perinatol 26:235-245

10. Fowlie PW, Davis PG 2003 Prophylactic indomethacin for preterm infants: a systematic review and meta-analysis. Arch Dis Child Fetal Neonatal Ed 88:F464F466

11. Ment LR, Vohr B, Oh W, Scott DT, Allan WC, Westerveld M, Duncan CC, Ehrenkranz RA, Katz KH, Schneider KC, Makuch RW 1996 Neurodevelopmental outcome at 36 months' corrected age of preterm infants in the Multicenter Indomethacin Intraventricular Hemorrhage Prevention Trial. Pediatrics 98:714-718

12. Couser RJ, Hoekstra RE, Ferrara TB, Wright GB, Cabalka AK, Connett JE 2000 Neurodevelopmental follow-up at 36 months' corrected age of preterm infants treated with prophylactic indomethacin. Arch Pediatr Adolesc Med 154:598-602

13. Schmidt B, Davis P, Moddemann D, Ohlsson A, Roberts RS, Saigal S, Solimano A, Vincer M, Wright LL 2001 Long-term effects of indomethacin prophylaxis in extremely-low-birth-weight infants. N Engl J Med 344:1966-1972
14. Dieni S, Inder T, Yoder B, Briscoe T, Camm E, Egan G, Denton D, Rees S 2004 The pattern of cerebral injury in a primate model of preterm birth and neonatal intensive care. J Neuropathol Exp Neurol 63:1297-1309

15. Coalson JJ, Winter VT, Siler-Khodr T, Yoder BA 1999 Neonatal chronic lung disease in extremely immature baboons. Am J Respir Crit Care Med 160:1333-1346

16. Yoder BA, Siler-Khodr T, Winter VT, Coalson JJ 2000 High-frequency oscillatory ventilation: effects on lung function, mechanics, and airway cytokines in the immature baboon model for neonatal chronic lung disease. Am J Respir Crit Care Med 162:1867-1876

17. McCurnin D, Seidner S, Chang LY, Waleh N, Ikegami M, Petershack J, Yoder B, Giavedoni L, Albertine KH, Dahl MJ, Wang ZM, Clyman RI 2008 Ibuprofeninduced patent ductus arteriosus closure: physiologic, histologic, and biochemical effects on the premature lung. Pediatrics 121:945-956

18. Gundersen HJ, Jensen EB 1987 The efficiency of systematic sampling in stereology and its prediction. J Microsc 147:229-263

19. Rees SM, Camm EJ, Loeliger M, Cain S, Dieni S, McCurnin D, Shaul PW, Yode B, McLean C, Inder TE 2007 Inhaled nitric oxide: effects on cerebral growth and injury in a baboon model of premature delivery. Pediatr Res 61:552-558

20. Rees S, Stringer M, Just Y, Hooper SB, Harding R 1997 The vulnerability of the fetal sheep brain to hypoxemia at mid-gestation. Brain Res Dev Brain Res 103:103118

21. Loeliger M, Watson CS, Reynolds JD, Penning DH, Harding R, Bocking AD, Rees SM 2003 Extracellular glutamate levels and neuropathology in cerebral white matter following repeated umbilical cord occlusion in the near term fetal sheep. Neurosci 116:705-714

22. Rees SM, Loeliger MM, Munro KM, Shields A, Dalitz PA, Dieni S, Thomson MA, Coalson J, Inder T 2009 Cerebellar development in a baboon model of preterm delivery: impact of specific ventilatory regimes. J Neuropathol Exp Neurol 68:605615

23. Loeliger M, Inder TE, Dalitz PA, Cain S, Camm EJ, Yoder B, McCurnin D, Shau PW, Clyman R, Rees SM 2009 Developmental and neuropathological consequences of ductal ligation in the preterm baboon. Pediatr Res 65:209-214

24. Loeliger M, Inder T, Cain S, Ramesh RC, Camm E, Thomson MA, Coalson J, Rees SM 2006 Cerebral outcomes in a preterm baboon model of early versus delayed nasal continuous positive airway pressure. Pediatrics 118:1640-1653

25. Loeliger M, Inder TE, Shields A, Dalitz P, Cain S, Yoder B, Rees SM 2009 High-frequency oscillatory ventilation is not associated with increased risk of neuropathology compared with positive pressure ventilation: a preterm primate model. Pediatr Res 66:545-550

26. Jaillard S, Larrue B, Rakza T, Magnenant E, Warembourg H, Storme L 2006 Consequences of delayed surgical closure of patent ductus arteriosus in very premature infants. Ann Thorac Surg 81:231-234 